\title{
Characterization of glycoconjugates in the endometrium of the miniature pig during different reproductive periods
}

\author{
Z. X. Zhou, Z. P. Deng and J. L. Chen \\ Department of Histology and Embryology, College of Veterinary Medicine, Beijing Agricultural \\ University, Beijing 100094, People's Republic of China
}

\begin{abstract}
Lectin-binding glycoconjugates in the endometrium of miniature pigs were determined for sexually immature, dioestrus, oestrus and early pregnancy periods, using five horseradish peroxidase-labelled lectins. Apparent changes associated with sexual maturity were increases in binding intensity of wheat germ agglutinin (WGA), Glycine max agglutinin (SBA) and Ulex europaeus agglutinin (UEA) and the appearance of Ricinus communis agglutinin (RCA) binding. The binding intensity of WGA, SBA and UEA further increased at oestrus, particularly for UEA which showed a stage-specific binding on the surface of endometrial epithelium. Pregnancy-related changes included an increase in WGA binding and a decrease in UEA and RCA binding. The results indicate that stage-specific alterations in lectin binding glycoconjugates occur in the endometrium of pigs associated with sexual maturity and the reproductive cycle.
\end{abstract}

\section{Introduction}

It is well known that the receptivity of the uterus to the implanting blastocyst and the attachment of the trophoblast to the endometrium are essential for implantation. Although the molecular mechanism of trophectoderm-epithelial interaction are unclear, the cell membrane glycoconjugates probably play an important role in this process, as cell surface carbohydrate has been implicated in cellular interactions such as cell-cell recognition, adhesion, fusion and differentiation (Knudsen $e t$ al., 1981; Damsky et al., 1982; Brandly and Schnaar, 1986; Edelman, 1986).

Many investigations have been made on pregnancy-related changes of lectin binding glycoconjugates in the endometrium of mice (Lee et al., 1983; Wu et al., 1983, 1991; Chavez and Anderson, 1985), rabbits (Anderson et al., 1986), cattle (Munson et al., 1989), pigs (Whyte and Robson, 1984), horses (Whyte and Allen, 1985) and humans (Jansen et al., 1985; Lee and Damjanov, 1985). These data indicate that the pre- and post-implantation processes are associated with changes in glycoconjugates in the endometrium. Furthermore, Lindenberg et al. (1988) demonstrated that a specific fucosylated pentasaccharide on the endometrial surface is involved in the initial attachment of the blastocyst to the endometrial epithelium of mice.

The expression of the glycoconjugates in the endometrium has been shown to be modulated by steroid hormones (Kimber and Lindenberg, 1990). Administration of exogenous hormones could alter the characterization of glycoconjugates in the endometrium and result in the degeneration of conceptuses (Blair et al., 1991; Kramber, 1992). However, little attention has been paid to the characterization of glycoconjugates in the

Received 4 May 1993 endometrium associated with sexual maturity and oestrus under normal hormonal regulation.

In the present study, five lectins, labelled with horseradish peroxidase (HRP), were used to determine the glycoconjugates in the endometrium of miniature pigs at different reproductive periods to obtain a better understanding of stage-specific changes. The relationship between endometrial glycoconjugates and possible uterine receptivity was also investigated.

\section{Materials and Methods}

Tissues

Miniature pigs used in this study were sexually immature (two months), in dioestrus, in oestrus or in early pregnancy (25 days). Four pigs were selected for each period. These animals were housed in pens with concrete floors and fed formula diet based on corn and soybean meal. The average body weight was 4.6 and $34.5 \mathrm{~kg}$ for sexually immature and adult animals, respectively. The period of oestrus was defined by examination of the vulva for swelling and reddening together with the exhibition of a standing oestrus reflex. One week after the onset of oestrus the pigs were allocated to the dioestrous group. When sampling, the two periods were further confirmed by checking for the presence of mature follicles at oestrus and pink corpora lutea at dioestrus. Tissue samples were taken from the uterine horn and fixed with Bouin's solution. Tissues were embedded in paraplast and sectioned at $5 \mu \mathrm{m}$ intervals.

\section{Lectin histochemistry}

The five HRP-labelled lectins - wheat germ agglutinin (WGA), Glycine max agglutinin (SBA), Ricinus communis 
Table 1. Lectins used and corresponding inhibitory sugars

\begin{tabular}{lcc}
\hline Lectins & Source & Inhibitory sugars \\
\hline WGA & Triticum vulgare (wheat germ) & Glycine max (soybean) \\
SBA & Ricinus communis & N-acetyl-D-glucosamine \\
RCA & Ulex europaeus & $N$-acetyl-D-galactosamine \\
UEA I & Canavalia ensiformis & D-galactose \\
Con A & & $\alpha$-L-fucose \\
& & Methyl $\alpha$-D-mannopyranoside \\
\hline
\end{tabular}

Table 2. Lectin binding sites on the endometrium of miniature pigs

\begin{tabular}{|c|c|c|c|c|}
\hline & \multicolumn{2}{|c|}{ Endometrial epithelium } & \multicolumn{2}{|c|}{ Endometrial gland } \\
\hline & Surface & Cytoplasm & Surface & Cytoplasm \\
\hline \multicolumn{5}{|l|}{ WGA } \\
\hline Sexually immature & + & - & ++ & ++ \\
\hline Dioestrus & +++ & ++ & +++ & +++ \\
\hline Oestrus & ++++ & +++ & ++++ & +++ \\
\hline Early pregnancy & ++++ & ++++ & +++ & ++ \\
\hline \multicolumn{5}{|l|}{ SBA } \\
\hline Sexually immature & + & - & \pm & - \\
\hline Dioestrus & ++ & - & + & - \\
\hline Oestrus & +++ & \pm & +++ & \pm \\
\hline Early pregnancy & +++ & ++ & + & \pm \\
\hline \multicolumn{5}{|l|}{$\mathrm{RCA}$} \\
\hline Sexually immature & \pm & - & \pm & - \\
\hline Dioestrus & +++ & - & \pm & - \\
\hline Oestrus & +++ & - & \pm & - \\
\hline Early pregnancy & + & - & - & - \\
\hline \multicolumn{5}{|l|}{ UEA } \\
\hline Sexually immature & \pm & - & \pm & - \\
\hline Dioestrus & \pm & - & ++ & - \\
\hline Oestrus & ++++ & \pm & +++ & +++ \\
\hline Early pregnancy & - & - & ++ & \pm \\
\hline \multicolumn{5}{|l|}{ Con $A$} \\
\hline Sexually immature & ++ & ++ & ++ & ++ \\
\hline Dioestrus & ++ & ++ & ++ & ++ \\
\hline Oestrus & + & + & + & + \\
\hline Early pregnancy & + & + & \pm & \pm \\
\hline
\end{tabular}

- : negative; \pm : trace; + : weak; ++ : moderate; +++ : strong; ++++ : very strong.

WGA: wheat germ agglutinin; SBA: Glycine max agglutinin; RCA: Ricinus communis agglutinin; UEA: Ulex europaeus agglutinin; Con A: concanavalin A.

agglutinin (RCA), Ulex europaeus agglutinin (UEA) and concanavalin A (Con A) - and the corresponding inhibitory sugars were purchased from Sigma Chemical Co. (St Louis, $\mathrm{MO}$ ). Details of these lectins and sugars are presented in Table I.

For lectin binding, tissue sections were processed through the following steps at room temperature: (I) Hydrogen peroxide ( $1 \%)$ in phosphate-buffered saline (PBS) $\left(0.01 \mathrm{~mol}^{-1}\right.$, $\mathrm{pH} 7.2$; Kuhlmann, 1984) for $15 \mathrm{~min}$; (2) three rinses in PBS; (3) addition of BSA for $30 \mathrm{~min}$; (4) three rinses in PBS; (5) incubation with the solution of HRP-labelled lectin in a moist chamber for $I h$; (6) three rinses in PBS; (7) incubation with $0.05 \%$ diaminobenzidine $(\mathrm{DAB})$ and $0.1 \%$ hydrogen peroxide in Tris buffer $\left(0.05 \mathrm{~mol} \mathrm{l}^{-1}, \mathrm{pH} 7.6\right)$ to visualize the bound sites; (8) dehydration through graded ethanol, clearing in xylene and mounting in resinous medium. Controls included (1) omitting HRP-labelled lectin, and (2) preabsorbing lectin with the appropriate inhibitory sugar $\left(0.2 \mathrm{~mol}^{-1}\right)$.

\section{Results}

The lectin-binding patterns in the endometrium at different reproductive periods are summarized (Table 2 ) and illustrated (Figs I-4). Generally, the lectin binding sites were on the apical surface or in the cytoplasm of the endometrial epithelial cells, and on the apical surface or in the supranuclear cytoplasm of the endometrial glandular cells. In control sections, the binding of WGA, SBA, RCA, UEA and Con A could be blocked by the addition of the corresponding inhibitory sugars (listed in Table 1). 

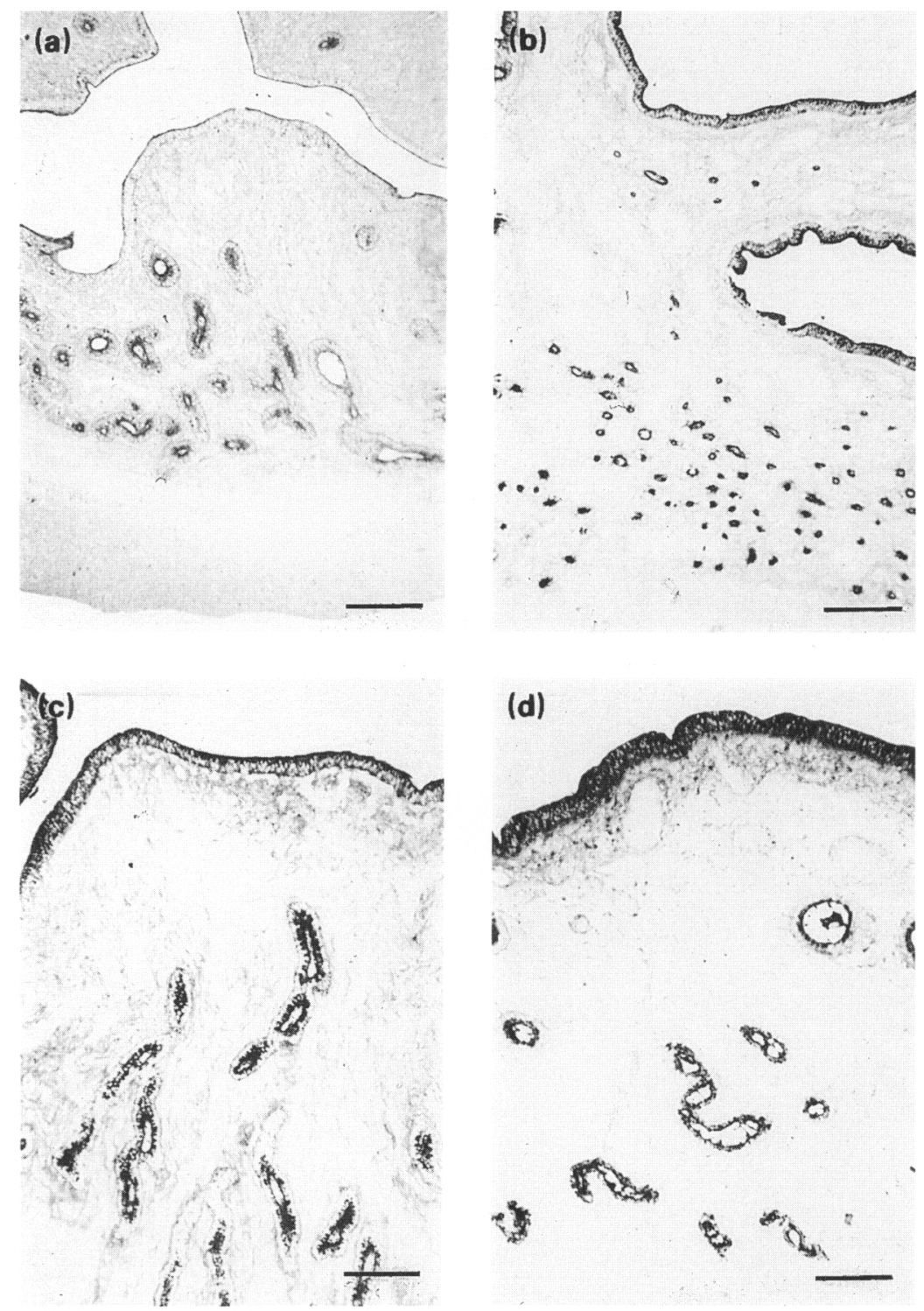

Fig. 1. Binding patterns of wheat germ agglutinin (WGA) on the endometrium in (a) sexually immature, (b) dioestrus, (c) oestrus and (d) early pregnancy. (a) There is weak binding on the endometrial epithelium and moderate binding on the endometrial gland. (b) Both endometrial epithelium and endometrial gland show strong binding. (c) The binding intensity is very strong on both endometrial epithelium and endometrial gland. (d) Note the increase in binding intensity on the cytoplasm of the endometrial epithelium. Scale bars represent $100 \mu \mathrm{m}$.

\section{WGA binding}

In the sexually immature animals, WGA binding was weak on the surface of the endometrial epithelium and moderate on the surface and in the cytoplasm of endometrial glands (Fig. 1a). At dioestrus, the binding on the surface of both endometrial epithelium and gland was strong, and the cytoplasm of endometrial epithelium also showed moderate WGA binding (Fig. 1b). The binding at oestrus increased to very strong on the surface and in the cytoplasm of both endometrial epithelium and gland (Fig. Ic). Compared with oestrus, stronger binding on the endometrial epithelium but weaker binding on the endometrial gland were found in early pregnancy (Fig. Id).

\section{$S B A$ binding}

SBA-binding sites in the sexually immature animals were present only on the surface of both the endometrial epithelium and gland, and the intensity was greater on the former and weaker on the latter (Fig. 2a). During dioestrus the binding intensity on the surface of endometrial epithelium and gland was moderate and weak, respectively (Fig. 2b). The binding intensity on the surface of both endometrial epithelium and 

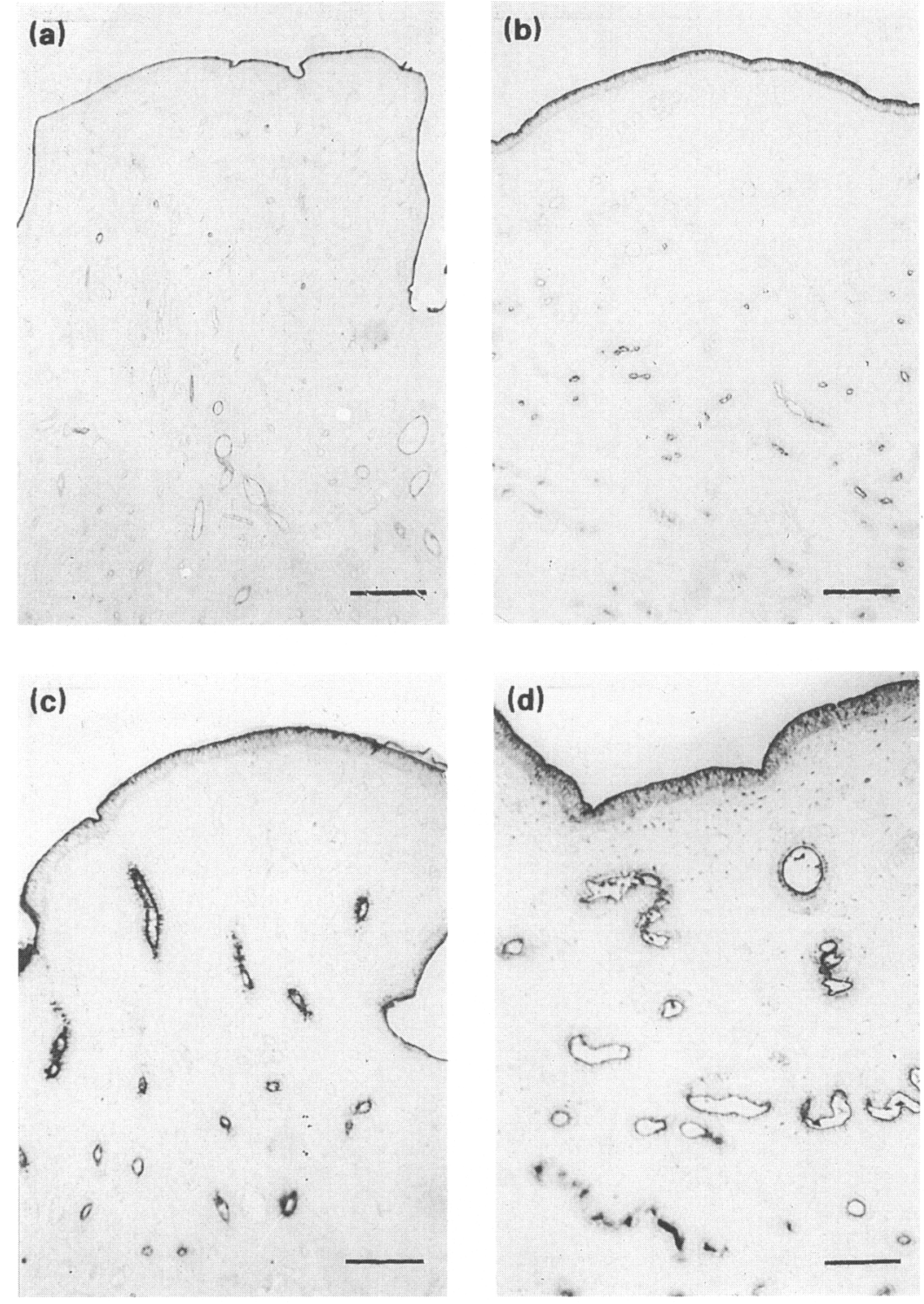

Fig. 2. Binding patterns of Glycine max agglutinin (SBA) on the endometrium in (a) sexually immature, (b) dioestrus, (c) oestrus and (d) early pregnancy. (a) The endometrial epithelium and gland show weak and trace binding respectively. (b) The binding intensity is moderate on the endometrial epithelium and weak on the endometrial gland. (c) Both endometrial epithelium and epithelial gland show strong binding. (d) An increase in binding intensity is seen on the cytoplasm of the endometrial epithelium. Scale bars represent $100 \mu \mathrm{m}$.

gland increased to strong at oestrus (Fig. 2c). In early pregnancy the endometrial epithelium had strong SBA binding on the surface and also showed moderate binding in the cytoplasm, while the binding on endometrial gland was lower (Fig. 2d).

\section{RCA binding}

The surface of both endometrial epithelium and gland showed very weak RCA binding in sexually immature animals (Fig. 3a). During the dioestrus period the binding on the surface of endometrial epithelium increased to strong, while the endometrial gland had very weak binding (Fig. $3 b$ ). The binding pattern at oestrus (Fig. 3c) was similar to that at dioestrus. In early pregnancy the RCA binding on the surface of endometrial epithelium was weak, and there was no binding on the endometrial glands (Fig. 3d).

\section{UEA binding}

In sexually immature animals, UEA binding was very weak and limited to the surface of endometrial epithelium and gland (Fig. 4a). At dioestrus the binding on the surface of the endometrial gland, but not on the epithelium, increased to 

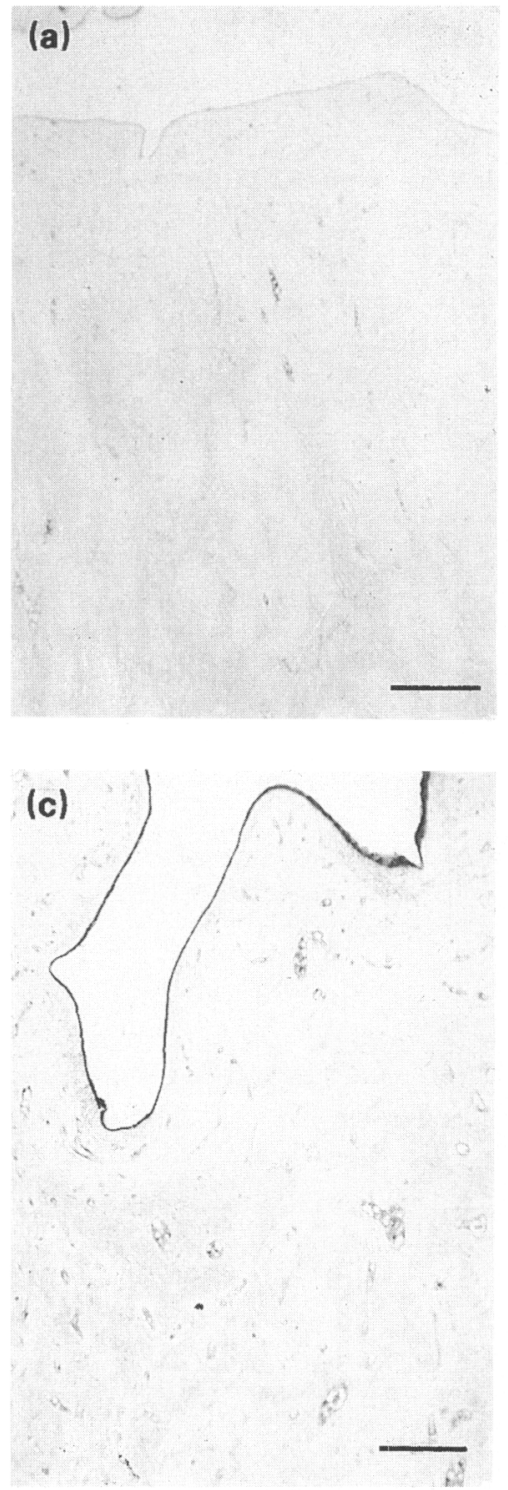

(b)

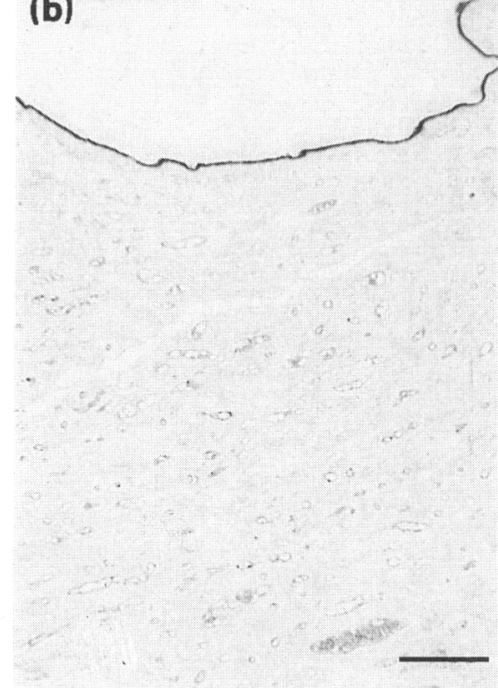

(d)

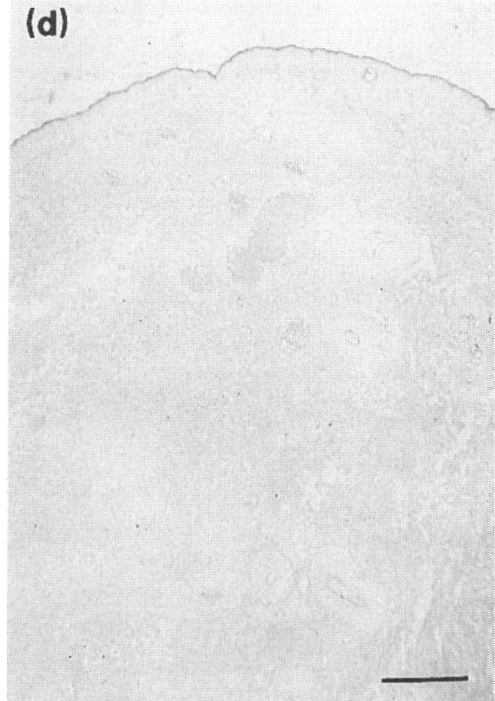

Fig. 3. Binding patterns of Ricinus communis agglutinin (RCA) on the endometrium in (a) sexually immature, (b) dioestrus, (c) oestrus and (d) early pregnancy. (a) Both endometrial epithelium and endometrial gland are almost unreactive. (b) The binding intensity is strong on the endometrial epithelium but very weak on the endometrial gland. (c) There is no stage-specific change. (d) Both the endometrial epithelium and endometrial gland show a decrease in binding intensity. Scale bars represent $100 \mu \mathrm{m}$.

moderate or strong (Fig. 4b). At oestrus, the binding increased to very strong on the surface of both the endometrial epithelium and gland, and the cytoplasm of some of the endometrial glands was also intensively stained (Fig. 4c). However, in early pregnancy there was no UEA binding on the surface of endometrial epithelium and that on the endometrial gland was also markedly decreased (Fig. 4d).

\section{Concanavalin A binding}

Both endometrial epithelium and gland had moderate Con A binding in sexually immature animals. No apparent changes were observed with sexual maturity, although at oestrus and in early pregnancy, the binding intensity showed a gradual decrease, especially on the endometrial gland.

\section{Discussion}

Although the pregnancy-related changes in lectin binding patterns on the endometrium have been demonstrated, little is known about the alterations associated with sexual maturity and during the oestrous cycle. Results presented here provide evidence of stage-specific changes of lectin binding on the 

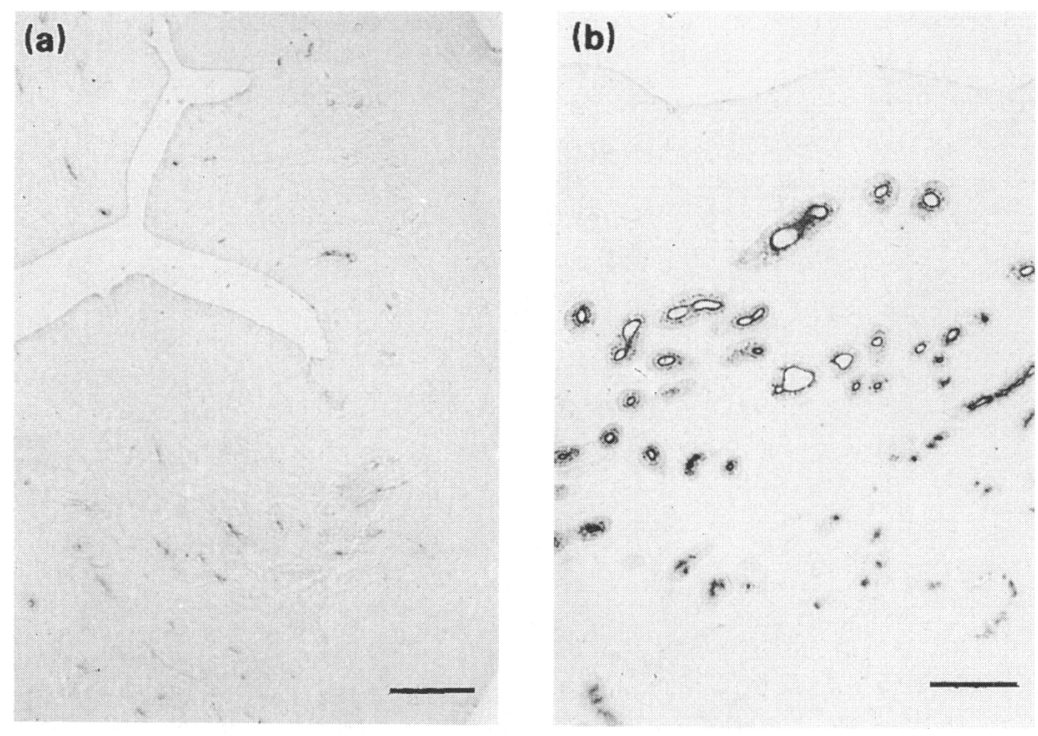

(c)
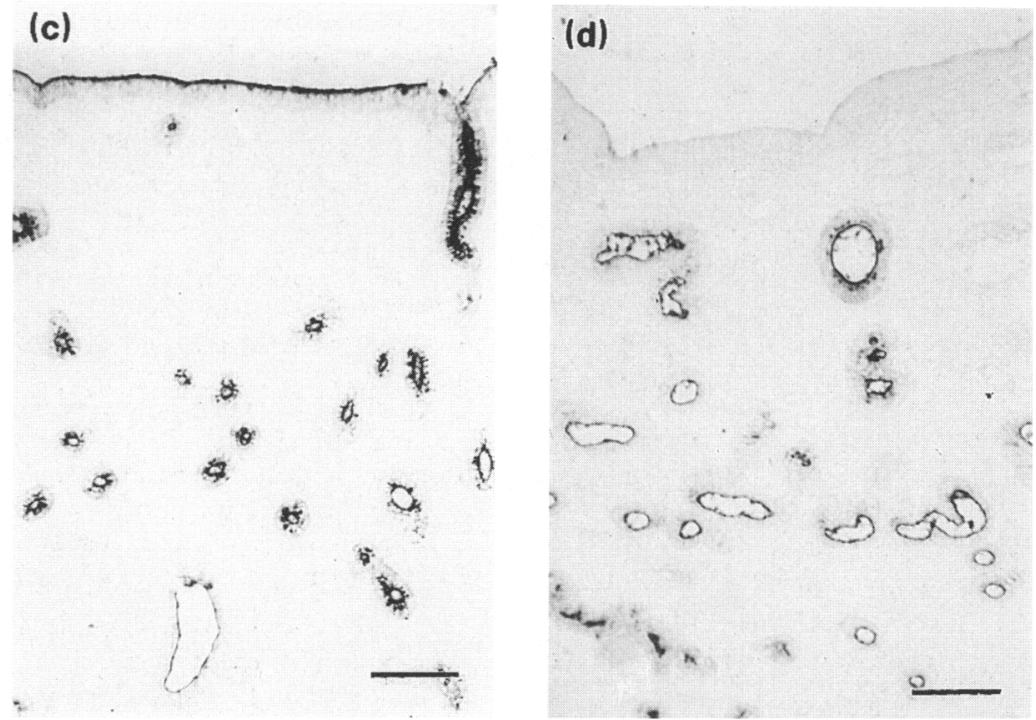

Fig. 4. Binding patterns of Ulex europaeus agglutinin (UEA) on the endometrium in (a) sexually immature, (b) dioestrus, (c) oestrus and (d) early pregnancy. (a) Only trace binding is seen. (b) The binding intensity increased to moderate on the endometrial gland. (c) Note the appearance of strong binding on the endometrial epithelium. (d) There is no binding on the endometrial epithelium. Scale bars represent $100 \mu \mathrm{m}$.

endometrium associated with sexual maturity and oestrus, as well as with pregnancy.

Changes in lectin binding pattern on the endometrium, associated with sexual maturity, have been demonstrated in the present study, including increases in reactivity of WGA, $\mathrm{SBA}$ and UEA and the appearance of RCA binding. These results indicate that both the quantity and pattern of glycoconjugates in the endometrium change relative to sexual maturity. These changes probably result from stimulation by sex hormones, as previous reports have shown that oestrogen stimulates the synthesis of glycoconjugates in the endometrium of some mammals (Kuivanen and DeSombre, 1985; Teng et al., 1986; Pentecost and Teng, 1987; Kimber and Lindenberg, 1990).
Among the five lectins used in the present study, the binding patterns of WGA, SBA and UEA showed apparent stage-specific changes. The binding intensities of the three lectins were stronger in both endometrial epithelium and endometrial gland at oestrus compared with dioestrus, particularly in the cytoplasm. The increased cytoplasmic binding may reflect active secretion by the endometrium. Alternatively, the binding of UEA to the surface of endometrial epithelium was specific for the stage of the oestrous cycle. Because glycoconjugates in the endometrial epithelium have been shown to be involved in the initial adhesion of the blastocyst to the endometrial epithelium (Jansen $e t$ al., 1985; Lindenberg et al., 1988), the appearance of UEA on the endometrial epithelial surface at oestrus suggests that the UEA binding 
glycoconjugate may be related to preparation of the uterine endometrium before implantation.

The pregnancy-associated alterations in lectin binding patterns observed in the present study include mainly increased WGA binding and decreased binding of RCA and UEA on the endometrial epithelium. The increase of WGA binding to the endometrial epithelium was similar to that reported for most other animal species (Lee et al., 1983; Whyte and Robson, 1984; Whyte and Allen, 1985; Anderson et al., 1986; Munson et al., 1989; Wu et al., 1991), although WGA binding did not change in the endometrium of pregnant women (Lee and Damjanov, 1985). The decrease of UEA binding also agrees with findings in mice (Lee et al., 1983; Wu et al., 1991), cattle (Munson et al., 1989) and women (Lee and Damjanov, 1985), but this result is contrary to the report for the horse (Whyte and Allen, 1985). Our observations on the pregnancy-related change of RCA binding are not in agreement with reports of increased binding in mice (Lee et al., 1983; Chavez and Anderson, 1985; Wu et al., 1991; Horvat, 1993) and rabbits (Anderson et al., 1986), and of no change in binding in cows (Munson et al., 1989) and humans (Lee and Damjanov, 1985). In the present study, lectin binding to the endometrial gland during pregnancy was weaker than during oestrus, but stronger than during dioestrus. This finding is in agreement with the finding of increased lectin binding on the endometrial gland in pregnant versus nonpregnant cattle (Munson et al., 1989).

Differences in lectin binding between the endometrial epithelium and endometrial gland were found in the study reported here. The limited binding of RCA to the surface of endometrial epithelium indicates that the corresponding glycoconjugate is a structural component of the endometrial epithelium, but is not a secretory component. This result differs from the positive staining of RCA in the endometrial glands of cows (Munson et al., 1989) and women (Lee and Damjanov, 1985). Contrary to RCA, UEA was mainly bound to the endometrial gland in miniature pigs, whereas UEA binding was totally absent in cows (Munson et al., 1989) and women (Lee and Damjanov, 1985; Yen et al., 1986). The discrepancy in UEA binding on the endometrial glands in different species may reflect a species difference in the composition of uterine secretions. The binding patterns of WGA, SBA and Con A to the endometrial epithelium and endometrial glands noted in this study are similar to those in other species (Lee and Damjanov, 1985; Yen et al., 1986; Munson et al., 1989).

In summary, the present study shows that the binding patterns of lectins on the endometrial epithelium and gland of miniature pigs change during the reproductive cycle. These changes suggest that the glycoconjugates in the endometrium are related to receptivity of the uterus for the implanting blastocyst. On the basis of these data, future studies will include the isolation of glycoproteins from the endometrium, using lectin affinity chromatography, to determine their significance in the interaction between the endometrium and trophoblast.

\section{References}

Anderson TL, Olson GE and Hoffman LH (1986) Stage-specific alterations in the apical membrane glycoprotein on endometrial epithelial cells related to implantation in rabbits Biology of Reproduction 34 701-720
Blair RM, Geisert RD, Zavy MT, Yellin T, Fulton RW and Short EC (1991) Endometrial surface and secretory alterations associated with embryonic mortality in gilts administered estradiol valerate on days 9 and 10 of gestation Biology of Reproduction 44 1063-1079

Brandly BK and Schnaar RL (1986) Cell-surface carbohydrates in cell recognition and response Journal of Leukocyte Biology 40 97-111

Chavez DJ and Anderson TL (1985) The glycocalyx of the mouse uterine Iuminal epithelium during estrus, early pregnancy, the peri-implantation period, and delayed implantation. I. Acquisition of Ricinus communis I binding sites during pregnancy Biology of Reproduction 32 1135-1142

Damsky CH, Knudsen KA and Buck CA (1982) Integral membrane glycoproteins related to cell-substratum adhesion in mammalian cells Journal of Cell Biochemistry 18 1-13

Edelman GM (1986) Cell adhesion molecules in the regulation of animal form and tissue pattern Annual Review of Cell Biology 2 81-116

Horvat B (1993) Galactose-binding lectins as markers of pregnancy-related glycoproteins Histochemistry 99 95-101

Jansen APS, Turner M, Johannisson E, Landgren B-M and Diczfalusy E (1985) Cyclic changes in human endometrial surface glycoproteins: a quantitative histochemical study Fertility and Sterility 44 85-91

Kimber SJ and Lindenberg S (1990) Hormonal control of a carbohydrate epitope involved in implantation in mice Joumal of Reproduction and Fertility $\mathbf{8 9}$ 13-21

Knudson KA, Rao PE, Damsky CH and Buck CA (1981) Membrane glycoproteins involved in cell-substratum adhesion Proceedings of the National Academy of Sciences, USA 78 6071-6075

Kramber B (1992) The effect of exogenous gonadotropins on the glycocalyx of the rat endometrium. In Abstracts of the Second International Conference of China on Anatomical Sciences. October 12-15, Beijing, China, pp 212

Kuhlmann WD (1984) Practical aspects. In Immunoenzyme Techniques in Cytochemistry, pp 137-155. Verlag Chemie, Weinheim; Deerfield Beach, Florida/Basel

Kuivanen PC and DeSombre ER (1985) The effect of sequential administration of $17 \beta$-estradiol on the synthesis and secretion of specific proteins in the immature rat uterus Journal of Steroid Biochemistry 22 439-451

Lee MC and Damjanov I (1985) Pregnancy-related changes in the human endometrium revealed by lectin histochemistry Histochemistry 82 275-280

Lee MC, Wu TC, Wan YJ and Damjanov I (1983) Pregnancy-related changes in the mouse oviduct and uterus revealed by differential binding of fluoresceinated lectins Histochemistry $\mathbf{7 9} 365-375$

Lindenberg S, Sundberg K, Kimber SJ and Lundblad A (1988) The milk oligosaccharide, lacto- $N$-fucopentaose $\mathrm{I}$, inhibits attachment of mouse blastocysts on endometrial monolayers Journal of Reproduction and Fertility 83 149-158

Munson L, Kao JJ and Schlafer DH (1989) Characterization of glycoconjugates in the bovine endometrium and chorion by lectin histochemistry Journal of Reproduction and Fertility 87 509-517

Pentecost BT and Teng CT (1987) Lactotransferrin is the major estrogen inducible protein of mouse uterine secretions Journal of Biological Chemistry 262 10134-10139

Teng CT, Walker MP, Bhattacharyya SN, Klapper DG, DiAugustine RP and McLachlan JA (1986) Purification and properties of an oestrogen-stimulated mouse uterine glycoprotein (approx. $70 \mathrm{kD}$ ) Biochemical Journal $\mathbf{2 4 0}$ 413-422

Whyte A and Allen WR (1985) Equine endometrium at pre-implantation stages of pregnancy has specific glycosylated regions Placenta 6 537-542

Whyte A and Robson T (1984) Saccharides localized by fluorescent lectins on trophectoderm and endometrium prior to implantation in pigs, sheep and equids Placenta 5 533-540

Wu TCJ, Wan YJ and Damjanov I (1983) Distribution of Bandeiraea simplicifolia lectin binding sites in the genital organs of female and male mice Histochemistry $77233-241$

Wu TCJ, Lee SM, Jih MH and Wan YJJ (1991) Distribution of lectin binding sites in the mouse oviduct and uterus during pregnancy Biology of Reproduction 44 (Supplement) 53

Yen Y, Lee MC, Salzmann M and Damjanov I (1986) Lectin binding sites on human endocervix: a comparison with secretory and proliferative endometrium Anatomical Record 215 262-266 